\title{
Enhancement of Visual Realism with BRDF for Patient Specific Bronchoscopy Simulation
}

\author{
Adrian J. Chung ${ }^{1}$, Fani Deligianni ${ }^{1}$, Pallav Shah ${ }^{2}$, Athol Wells ${ }^{2}$, and \\ Guang-Zhong Yang ${ }^{1}$ \\ 1 Department of Comupting, Imperial College, London \\ 2 Royal Brompton Hospital, London
}

\begin{abstract}
This paper presents a novel method for photorealistic rendering of the bronchial lumen by directly deriving matched shading and texture parameters from video bronchoscope images. 2D/3D registration is used to match video bronchoscope images with 3D CT scan of the same patient, such that patient specific modelling and simulation with improved visual realism can be achieved. With the proposed method, shading parameters are recovered by modelling the bidirectional reflectance distribution function (BRDF) of the visible surfaces by exploiting the restricted lighting configurations imposed by the bronchoscope. The derived $\mathrm{BRDF}$ is then used to predict the expected shading intensity such that a texture map independent of lighting conditions can be extracted. This allows the generation of new views not captured in the original bronchoscopy video, thus allowing free navigation of the acquired 3D model with enhanced photo-realism.
\end{abstract}

\section{Background}

With the maturity of minimal access surgery in recent years, there has been an increasing demand of patient specific simulation devices for both training and skills assessment. This is due to the fact that the complexity of the instrument controls, restricted vision and mobility, difficult hand-eye co-ordination, and the lack of tactile perception are major obstacles in performing minimal access surgeries. They require a high degree of manual dexterity from the operator. Computer simulation provides an attractive means of performing certain aspects of this training, particularly the hand eye co-ordination and instrument control. One significant challenge to computer based simulation is the creation of patient specific models combined with photo-realistic rendering so that basic as well as advanced surgical skills can be assessed with these simulation platforms.

For patient specific bronchoscope simulation, a number of techniques have been proposed for co-registering bronchoscope videos with $3 \mathrm{D}$ tomographic data such that camera pose in relation to the bronchial tree during video bronchoscope examination can be derived 12 With the use of image based modelling and rendering techniques, it is possible to extend conventional texture mapping to support the representation of 3D surface details and view motion parallax in addition to photorealism [3,4,5]. One of the major challenges of combining 2D 
video with $3 \mathrm{D}$ morphological data for patient specific simulation is the extraction of intrinsic surface texture and reflectance properties that is not dependent on specific viewing conditions. This allows the generation of new views with different camera and lighting configurations. For surgical simulation, this permits the incorporation of tissue instrument interaction, and thus greatly enhances the overall realism of the simulation environment. The purpose of this paper is to introduce a novel technique based on BRDF modelling for the recovery of intrinsic visual properties of the surface. An essential part of this process is the factoring of each video image into a surface shading function and texture map, as this enables new viewpoints to be visualised. With the effective use of $2 \mathrm{D} / 3 \mathrm{D}$ registration, we demonstrate how the proposed method can be used to generate new renditions that are morphologically accurate and visually photo-realistic during free navigation of the $3 \mathrm{D}$ model.

\section{Methods}

\subsection{Image Based Modelling of BRDF}

One method for rendering a realistic visualisation of a surface is to solve the complete Rendering Equation[6]:

$$
\begin{aligned}
L_{p}\left(\theta_{r}, \phi_{r}\right) & =E_{p}\left(\theta_{r}, \phi_{r}\right) \\
& +\int_{\theta_{i} \in\left[0, \frac{\pi}{2}\right]} \int_{\phi_{i} \in[0,2 \pi]} \rho_{p}\left(\theta_{i}, \phi_{i}, \theta_{r}, \phi_{r}\right) I_{p}\left(\theta_{i}, \phi_{i}\right) \cos \left(\theta_{i}\right) d \phi_{i} d \theta_{i}
\end{aligned}
$$

which states that the light exiting, $L$, from a point, $p$, in a direction given by spherical polar coordinates $\left(\theta_{r}, \phi_{r}\right)$, depends on light emitted by that point, $E_{p}$, and light reflected, $I_{p}$, from all incoming directions $\left(\theta_{i}, \phi_{i}\right)$ over the hemisphere. Light is not reflected equally from every direction however. It is weighted by a bidirectional reflectance distribution function, $\rho\left(\theta_{i}, \phi_{i}, \theta_{r}, \phi_{r}\right)$, whose properties are determined solely by the material being rendered. A variety of functions have been proposed for $\rho$ that cater for specific classes of materials [7]. These models usually have several parameters to adjust in order to match the characteristics of the target material, and there is often no robust way to ensure accuracy of the values chosen. For some models [8] optimisation techniques can be used for parameter estimation provided the reflectance of the target material can be measured over a range of illumination directions and intensities. This approach requires a great degree of control over lighting conditions to ensure adequate coverage of the high-dimensional domain over which $\rho$ is defined 9 . For this purpose, a special reflectance measurement apparatus can be specifically designed for the task 10. By extending this strategy to entire images rather than point measurements of reflectance, one can measure not only a single BRDF but an entire set of BRDFs and map them as a texture map over the surface of an object of known geometry [11.

In situations where one has little control over the lighting environment, an approach based on global illumination modelling may be pursued. Given images 
captured from a finite number of viewing positions, the outgoing light intensities for several points in the environment are extracted and then by inverting the Rendering Equation, the BRDFs are determined 1213. Normally a large number of images must be captured from a wide range of directions and viewpoints, however there are techniques which rely on the uniformity of the materials so that the number of input images can be significantly reduced [14]. In the case of bronchoscopy, one has limited control over the lighting conditions and the choice of viewpoints and viewing angle is restricted by the tubular structure of the airways. For this reason, a certain degree of uniformity of the reflectance in the bronchial lumen is assumed in subsequent sections. The overall steps involved in the proposed technique are illustrated in Figure 1 where 2D/3D registration 2] was applied to the $2 \mathrm{D}$ video and $3 \mathrm{D} \mathrm{CT}$ data sets after radial distortion correction of the video camera 15. Illumination parameters are extracted through BRDF and attenuation estimation, which is then used to recover the global texture map. The extracted surface texture and surface illumination characteristics allow the generation of new views of the $3 \mathrm{D}$ model by using the $3 \mathrm{D}$ mesh derived from the 3D CT images.

For this study, patients to undergo bronchoscope examination were scanned with a Siemens Somaton Volume Zoom 4-Channel Multidetector CT with a slice thickness of $3 \mathrm{~mm}$ and in-plane resolution of $1 \mathrm{~mm}$. The bronchial tree was segmented from the other anatomy using region growing [16] and morphological operators. Subsequently, the airway surface was reconstructed as a polygonal mesh by using the marching cubes algorithm. This mesh was then registered with the image frames captured from the video output of the bronchoscope and the end result was a pairing of video frames with camera poses within the bronchial airway from which each video image had been taken.

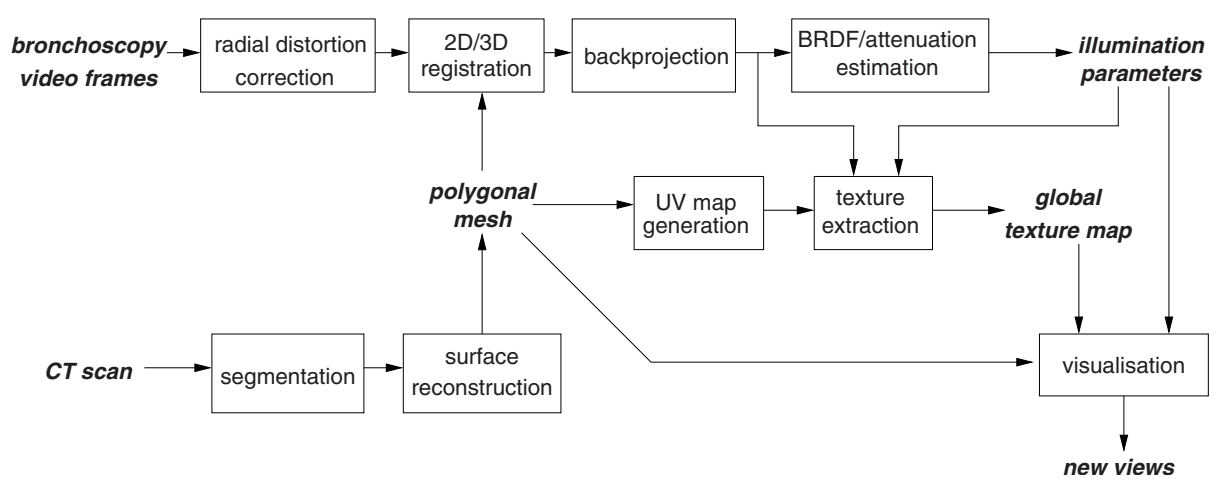

Fig. 1. The CT data and bronchoscopy video must pass through several stages of processing to create realistic patient specific visualisations from novel view points. 


\subsection{Simplified Bidirectional Reflectance Distribution Function}

In general, modelling the BRDF of any real world material requires the representation of a function over a parameter space of at least four dimensions, $\left(\theta_{i}, \phi_{i}, \theta_{r}, \phi_{r}\right)$. Fortunately the specific lighting configuration imposed by the bronchoscopy examination allows a number of simplifications to be made to the BRDF and the global illumination model.

- There is only one light source and it always coincides with the viewpoint. That is, $\theta_{i}=\theta_{o}$ and $\phi_{i}=\phi_{o}$.

- The inner airway surface is assumed to be isotropic. Thus, the BRDF is independent of $\phi_{i}$ and hence can be modelled as a function of $\theta_{i}$ only.

- The intensity of light incident on a surface depends mainly on distance from light source and the width of the airway.

By taking the above into consideration, the illumination conditions within the airway were modelled by using a cubic curve parameterised on the normalised scalar product of viewing vector $\boldsymbol{V}$ and surface normal $\boldsymbol{N}$.

$$
\rho_{p}(\boldsymbol{V}, \boldsymbol{N})=\sum_{0}^{3} c_{i} B_{i}^{3}(\gamma) \text { where } \gamma=\frac{\boldsymbol{V} \cdot \boldsymbol{N}}{|\boldsymbol{V}||\boldsymbol{N}|}, B_{i}^{n}(t)=\frac{n !}{i !(n-i) !}(1-t)^{n-i} t^{i}
$$

Variations in shade cannot all be accounted for by BRDF effects alone, as intensity also depends on distance from light source. This depth dependent variation observed from viewpoint, p, was also modelled using a cubic curve:

$$
\varrho_{p}(z)=B_{0}^{3}(r)+\sum_{1}^{3} d_{i} B_{i}^{3}(r)
$$

where $r=\left(z-z_{\min }\right) /\left(z_{\max }-z_{\min }\right)$ and $z$ is the distance of the surface point from the viewpoint. The linear shift of the parameter, $z$, was needed to avoid errors due to extrapolation. The shade of a surface point observed from viewpoint, $\mathbf{p}$, is thus:

$$
\Psi_{p}(\boldsymbol{V}, \boldsymbol{N}, z)=\rho_{p}(\boldsymbol{V}, \boldsymbol{N}) \varrho_{p}(z)
$$

\subsection{Back Projection and Parameter Estimation}

For each $\mathbf{p}$ there is a unique set of parameters, $\left(c_{0}, c_{1}, c_{2}, c_{3}, d_{1}, d_{2}, d_{3}\right)$, that determine the intensity of every visible point in the $3 \mathrm{D}$ model. These parameters were estimated by backprojecting each registered video image onto the 3D geometry and then fitting $\Psi$ to the pixel intensities. Using the position of the bronchoscope that was determined through $2 \mathrm{D} / 3 \mathrm{D}$ registration 1 , each pixel in the video image was backprojected onto the 3D geometry to estimate the surface normal and distance from viewpoint. The following cost function is then minimised over all pixels:

$$
C_{p}=\sum_{i} w_{i}\left(\Psi_{p}\left(\boldsymbol{V}_{i}, \boldsymbol{N}_{i}, z_{i}\right)-P_{i}\right)^{2}
$$


where $P_{i}$ is the pixel intensity. To ensure that $\Psi$ fits the sample points adequately in areas of low sample point density a weighting factor, $w_{i}$, was included to compensate for the non-uniform distribution of samples. A histogram was applied to the $(\gamma, z)$ domain and $w_{i}$ was set to the inverse of the estimated sample density.

\subsection{Texture Mapping and Generating New Views}

The polygonal mesh reconstructed from the CT data was used to generate a texture atlas 17] on which a global texture map was constructed. The texture was extracted from each video frame by dividing the pixel intensities by $\Psi\left(\gamma_{i}, z_{i}\right)$. Multiple textures were combined into a single map using weighted averaging. Weights were initially chosen based on the intensities predicted by the $\Psi$ function since low intensities often yielded unreliable texture values, however this yielded blurry textures. Instead, for each texture pixel the non-maximal weights were suppressed and the resulting weight map was smoothed in the texture domain. This yielded sharper textures while reducing discontinuities. To generate a new view from a view point, $\mathbf{p}_{n e w}$, the nearest point, $\mathbf{p}$, was found for which $\Psi_{p}$ had previously been estimated. The $3 \mathrm{D}$ geometry was raycast from $\mathbf{p}_{n e w}$, and the surface normal and depth information were converted to intensities using $\Psi_{p}$. This was then multiplied by texture values at corresponding points in the global texture map.
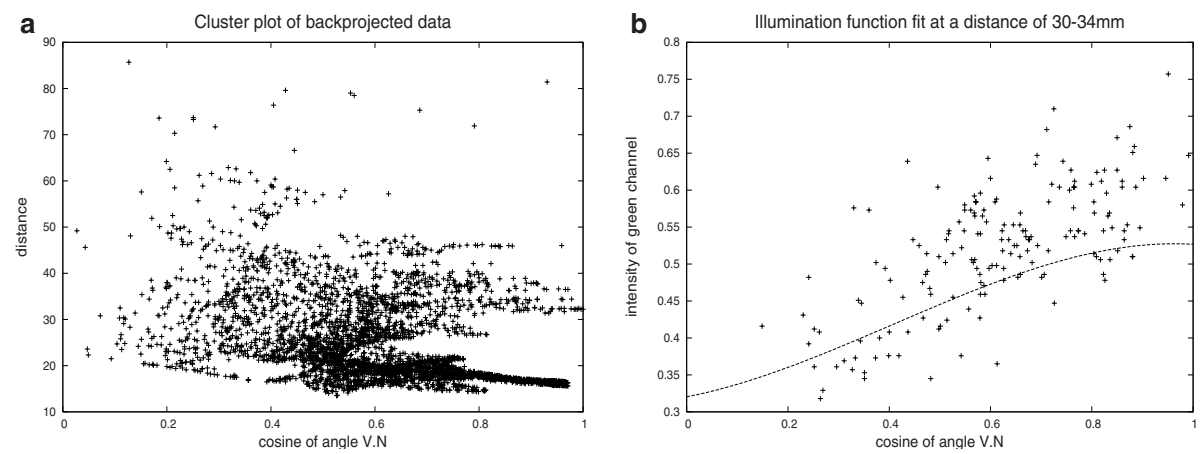

Fig. 2. (a) Cluster plot showing uneven distribution of backprojected samples. To ensure that Equation 4 fits the data over the entire domain and not just in regions of high density, each sample point was weighted inversely to point density. (b) The $\Psi$ function is shown fitting to the data at depth $30-34 \mathrm{~mm}$. The function must fit sample points at all depths.

\section{Results and Discussion}

To illustrate how $\Psi$ fits the sample points in areas of low sample density through the use of $w_{i}$, Figure 2 shows the cluster plot of the backprojected samples and 


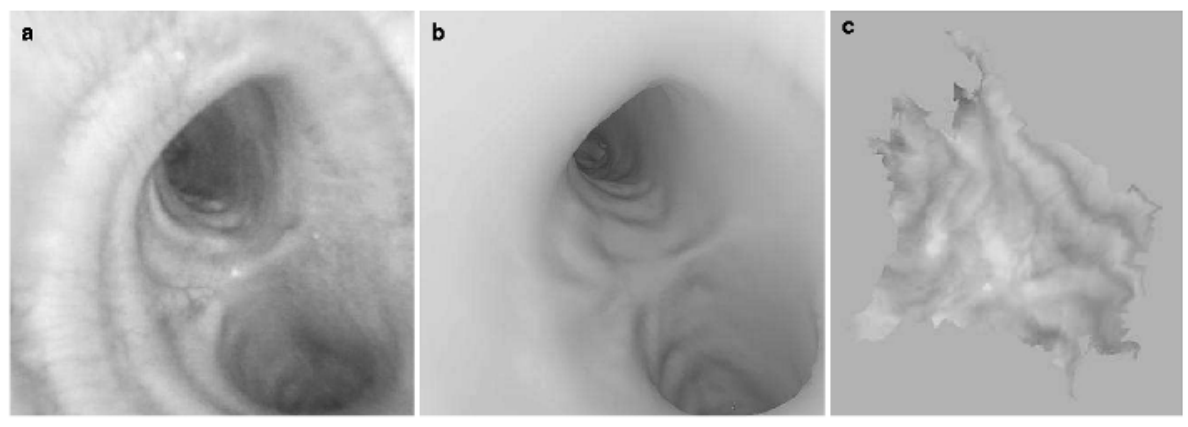

Fig. 3. (a) An original video frame has been corrected for radial distortion in preparation for $2 \mathrm{D} / 3 \mathrm{D}$ registration and backprojection. (b) The backprojected intensity values were used to estimate parameters for the $\Psi$. This illumination model was then used to predict the expected intensities of each pixel in the red, green, and blue channels. A part of the final extracted texture map is shown in (c) after remapping to the domain of the $2 \mathrm{D}$ texture map.

an example $2 \mathrm{D}$ plot showing the $\Psi$ function is fitted to the data at depth $30-34$ mm.

Figure 3(a) shows a video frame to which $\Psi$ has been fitted. The intensities predicted by $\Psi$ were mapped to the geometry when viewed from the same viewpoint as the bronchoscope (b). This was subsequently factored out of the original video frame, resulting in a texture independent of shading variations due to BRDF and global illumination.

To demonstrate how the extracted texture, which is independent of shading variations due to BRDF and global illumination, can be used to generate new views of the 3D structure, Figure 3(c) shows rendering results by using the proposed technique. It is evident that the rendered results retain the photorealism required despite the significant change in viewing angles.

In summary, we have presented a new technique based on BRDF modelling for recovering intrinsic surface properties of the bronchial tree. The method factorises each 2D video bronchoscope image into a surface shading function and texture map so that new views can be rendered with photo-realistic appearance. The method presented here exploits the restricted lighting configurations imposed by the bronchoscope, which significantly simplifies the use of BRDF to predict expected shading intensity so that a texture map independent of lighting conditions can be extracted. The current method assumes the initial $2 \mathrm{D} / 3 \mathrm{D}$ registration is accurate, but in reality this is difficult to guarantee particularly in the presence of airway deformation between pre-operative CT and bronchoscope examination. With the advent of miniaturised catheter electro-magnetic trackers, however, it is now possible to achieve relatively accurate camera-pose estimation with 5 degrees-of-freedom, thus significantly enhancing the accuracy of the registration process. 

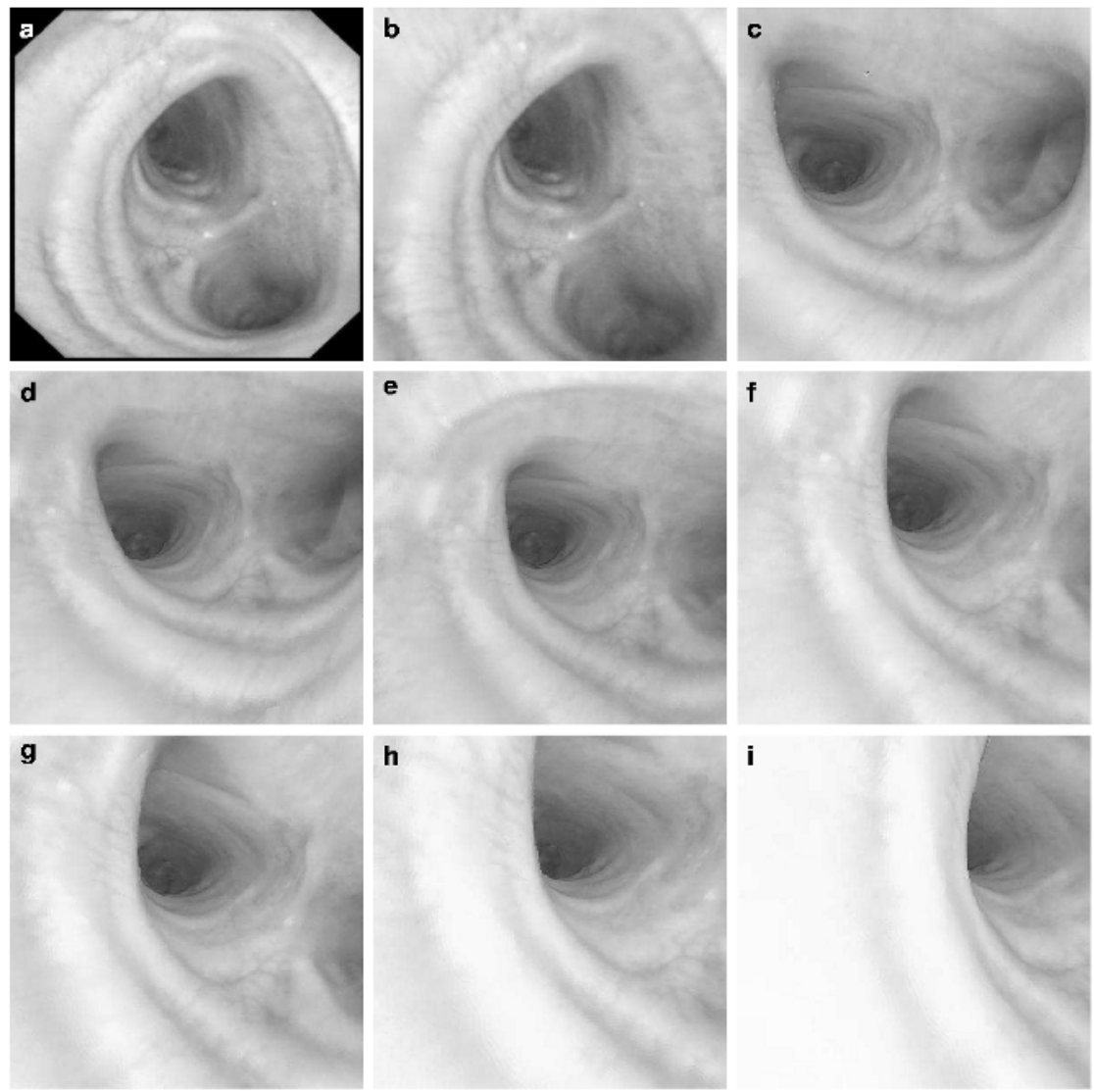

Fig. 4. (a)A typical frame captured from the video stream output of the bronchoscopy video processor is shown here. Radial distortion which must be corrected $\mathbf{b}$ prior to BRDF estimation. (c-i) New views of the bronchial lumen were generated using texture maps merged from the training video frames.

\section{References}

1. Mori, K., Deguchi, D., Sugiyama, J., Suenaga, Y., an d C. R. Maurer Jr., J.T., Takabatake, H., Natori, H.: Tracking of a bronchoscope using epipolar geometry analysis and intensity -based image registration of real and virtual endoscopic images. Medical Image Analysis 6 (2002) 321-336

2. Deligianni, F., Chung, A., Yang, G.Z.: pq-space 2d/3d registration for endoscope tracking. In: Conference on Medical Image Computing \& Computer Assisted Intervention (MICCAI03). Volume 1. (2003) 311-318

3. McMillan, L.: An image-based approach to three-dimensional computer graphics. Technical Report TR97-013 (1997)

4. Chen, S.E., Williams, L.: View interpolation for image synthesis. In: SIGGraph 1993. (1993) 279-288 
5. Debevec, P.E.: Pursuing reality with image-based modeling, rendering, and lighting. In: Second Workshop on 3D Structure from Multiple Images of Large-scale Environments and applications to Virtual and Augmented Reality (SMILE2), Dublin, Ireland. (2000)

6. Kajiya, J.T.: The rendering equation. In Evans, D.C., Athay, R.J., eds.: Computer Graphics (SIGGRAPH '86 Proceedings). Volume 20. (1986) 143-150

7. He, X.D., Torrance, K.E., Sillion, F.X., Greenberg, D.P.: A comprehensive physical model for light reflection. Computer Graphics 25 (1991) 175-186

8. Lafortune, E.P., Willems, Y.D.: Using the Modified Phong BRDF for Physically Based Rendering. Technical Report CW197, Department of Computer Science, Katholieke Universiteit Leuven, Leuven, Belgium (1994)

9. Ward, G.J.: Measuring and modeling anisotropic reflection. In Catmull, E.E., ed.: Computer Graphics (SIGGRAPH '92 Proceedings). Volume 26. (1992) 265-272

10. Dana, K.J., van Ginneken, B., Nayar, S.K., Koenderink, J.J.: Reflectance and texture of real-world surfaces. In: ACM Transactions on Graphics. Volume 18 (1). (1999) 1-34

11. Lensch, H.P.A., Kautz, J., Goesele, M., Heidrich, W., Seidel, H.P.: Image-based reconstruction of spatial appearance and geometric detail. ACM Transactions on Graphics 22 (2003) 234-257

12. Yu, Y., Debevec, P., Malik, J., Hawkins, T.: Inverse global illumination: Recovering reflectance models of real scenes from photographs. In Rockwood, A., ed.: Proceedings of the Conference on Computer Graphics (Siggraph99), N.Y., ACM Press (1999) 215-224

13. Loscos, C., Frasson, M.C., Drettakis, G., Walter, B., Grainer, X., Poulin, P.: Interactive virtual relighting and remodeling of real scenes. Available from www.imagis.imag.fr/Publications RT-0230, Institut National de Recherche en Informatique en Automatique (INRIA), Grenoble, France (1999)

14. Boivin, S., Gagalowicz, A.: Image-based rendering of diffuse, specular and glossy surfaces from a single image. In Fiume, E., ed.: SIGGRAPH 2001, Computer Graphics Proceedings. Annual Conference Series, ACM Press / ACM SIGGRAPH (2001) 107-116

15. Zhang, Z.: A flexible new technique for camera calibration. IEEE Transactions on Pattern Analysis and Machine Intelligence 22 (2000) 1330-1334

16. Schlathölter, T., Lorenz, C., Carlsen, I.C., Renisch, S., Deschamps, T.: Simultaneous segmentation and tree reconstruction of the airways for virtual bronchoscopy. In: Proceedings of SPIE, Medical Imaging 2002: Image Processing. Volume 4684. (2002) 103-113

17. Lévy, B., Petitjean, S., Ray, N., Maillot, J.: Least squares conformal maps for automatic texture atlas generation. In Spencer, S., ed.: Proceedings of the 29th Conference on Computer Graphics and Interactive Techniques (SIGGRAPH-02). Volume 21, 3 of ACM Transactions on Graphics., New York, ACM Press (2002) 362-371 